\title{
Stefan Horton
}

\section{Die ,Revolution der Hoffnung" und ihre Ergebnisse - Einige Bemerkungen zur gegenwärtigen Situation in Polen *)}

Die offene ökonomische und soziale Krise, die mit den Junistreiks der Arbeiter in den großen Industrieunternehmen von Ursus und Radom begann, konnte bis heute nicht gelöst werden. Das bedeutet, daß weder der sich an der Macht befindende Block noch die Arbeiterklasse mit den sie unterstützenden kleinen Gruppen der Intelligenz in der Lage sind, eine eindeutige politische Lösung durchzusetzen, um so die Bedingungen zu schaffen für die Beseitigung (oder deutliche Abschwächung) dieser Krise. Diese Unfähigkeit, in fast fünf Monaten ein politisches „Heilmittel“ zu finden, weist auf den schwerwiegenderen und tiefgehenderen Charakter der gegenwärtigen ökonomischen und sozialen Krise hin, die sich von den Krisen der 60er und 70er Jahre unterscheidet, die durch die Arbeiteraufstände in der Küstenregion bestimmt waren.

Die folgenden Bemerkungen stellen den Versuch einer einführenden Analyse der Hauptkennzeichen der gegenwärtigen Krise dar sowie einiger möglichen Lösungsformen.

\section{A. Momente der Krisensituation}

Wir werden zunächst auf die wichtigsten ökonomischen und sozialen Aspekte der derzeitigen Situation eingehen (1).

Auf der ökonomischen Ebene bedeutet die jetzige Krise das Scheitern des Entwicklungskonzepts infolge der

a) Differenzierung des Konsumgütermarkts zum Nachteil der Grundinteressen der Arbeiterklasse und zum Vorteil der oberen Partei- und Staatsbürokratie, der ,Arbeiteraristokratie" und der technokratischen Intelligenz;

b) beschleunigten Modernisierung der Industriestruktur durch Kredite und andere von den kapitalistischen Industrieländern ermöglichte Finanzierungsformen;

c) strukturellen Ungleichgewichte zwischen industriellem und landwirtschaftlichem Sektor;

d) Erhaltung des bürokratisch-zentralistischen Systems von Management und nationaler Wirtschaftsplanung;

e) kontrollierten Inflation.

*) Der Autor dieses Beitrages, für dessen Namen wir ein Pseudonym verwenden, lebt in Warschau. Das Manuskript wurde im November 1976 abgeschlossen. Übersetzung: H.H. Harbort

1 Eine ausführlichere Analyse einiger Aspekte der Krise enthält der vorstehende Aufsatz von Henryk Szlajfer, ,Nachzuholende Entwicklung unter Bedingungen des Weltmarkts". 
Die angeführten Faktoren sind Ausdruck sowohl langfristiger Tendenzen des ökonomischen Systems als auch von Phänomenen konjunktureller Art, wie sie als Folge der jeweiligen Wirtschaftspolitik auftraten. Im gegenwärtigen ,politischen“" Augenblick" interessiert uns besonders das Ausmał, in dem der Block an der Macht versucht, die mit den zunehmenden Anzeichen einer ungünstigen ökonomischen Konjunktur und langfristiger Prozesse verbundene politische Bedrohung zu neutralisieren, damit also das tatsächliche Ausmaß der (vom Block an der Macht) geforderten Kontrolle der ökonomischen Forderungen bestimmter Gesellschaftsklassen und -schichten, die eine Umsetzung in politische Forderungen verhindern soll.

Bevor wir darauf näher eingehen, sollten wir noch auf die sozialen Aspekte der gegenwärtigen Krise hinweisen, die sich $u$. a. ausdrücken in

a) einer weitreichenden Einkommens- und Besitzdifferenzierung sowohl zwischen als auch innerhalb einzelner gesellschaftlicher Klassen und Schichten;

b) einem Aufstieg einer ökonomisch-sozialen Interessengruppe, die (im objektiven Sinne) sehr an der Aufrechterhaltung dieser Differenzierung und der für sie vorteilhaften Produktionsstruktur interessiert ist (2);

c) einer relativen Verschlechterung der sozio-ökonomischen Situation des Hauptteils der Arbeiterklasse sowie anderer mittlerer und unterer urbaner Schichten (dies gilt fuir die 2. Hälfte des laufenden Fünfjahresplanes);

d) einer relativen Verschlechterung der sozio-ökonomischen Situation der Landbevölkerung, was sich vor allem in einer breiteren ,Einkommens-Kluft " zwischen Stadt und Dorf ausdrückt (die auch während des laufenden Fünfjahresplans anhält);

e) einer Verletzung des Prinzips der „sozialen Sicherheit“" durch den Mechanismus des Einkommens-Inflationsausgleichs und das Geschäft mit den gesellschaftlichen Konsumtionsgütern;

f) wachsenden Widersprïchen in den Unternehmen zwischen der Belegschaft und dem höheren Management (3);

g) einer Weigerung der Arbeiterklasse und der Einzelbauern, die Kosten für die Krisenfinanzierung und die makro-ökonomische Verschwendung zu bezahlen;

h) einer Zunahme von Elementen einer Innungs-Solidarität unter den Manufakturarbeitern, im Gegensatz zu offiziellen Vertretern und Bevollmächtigten (z. B. der Gewerkschaften);

2 Der einzige Versuch einer soziologischen Analyse diesen neuen Phänomens, der mir bekannt ist, ist Winicjusz Narojek's Spoleszestwo planujace. Proba socjologii gospodarki socjalistycznej (Eine Planungsgesellschaft. Ein Versuch einer Soziologie der sozialistischen Ökonomie), Warschau 1973. Narojek schreibt: ,Das von den in der sozialistischen Planwirtschaft Beschäftigten zur Schau gestellte Bemühen um Maximierung (des eigenen Anteils an der Konsumtion) unter gegebenen ökonomischen und organisatorischen Bedingungen ist in mehrfacher Hinsicht mit dem Verhalten der Klassen vergleichbar (. . .). In der sozialistischen Gesellschaft betrifft dieses Phänomen wahrscheinlich ausschließlich die Sphäre des ökonomischen Verhaltens im eigentlichen Sinn des Wortes (...) und es hat relativ geringe Aussichten zur Basis der politischen Artikulation zu werden. " (S. 287 - 288)

3 Eindeutige Beispiele finden sich in den Artikeln, die in den letzten Monaten in den Spalten der "Polityka"-Wochenschrift, vor allem in den Novemberausgaben veröffentlicht wurden, worin die Erfahrungen von Arbeitern der, Warschau"-Stahlwerke beschrieben werden. 
i) einer sozialen und politischen Aktivierung der Gruppen der städ tischen Intelligenz.

Alle angeführten ökonomischen und sozialen Faktoren traten schon mehr oder weniger ausgeprägt in den Krisen vor den Junistreiks auf, insbesondere im Dezember 1970. Ein Großteil von ihnen ist schließlich wesentliches Moment des herrschenden sozio-ökonomischen Systems. In diesem Sinne ist die gegenwärtige Krise nur eine modifizierte Form der vorhergehenden, wenn auch weit stärker ausgeprägt infolge der radikalen ökonomischen Maßnahmen in einigen Bereichen (z. B. Kreditpolitik, Außenhandelspolitik, Ausnutzung der Umverteilungseffekte der Inflation usw.)

Dieser Radikalismus, der manchmal wie ein Vabanque-Spiel erscheint, ist nichts anderes als der verzweifelte Versuch, den ökonomischen Stillstand zu bekämpten, ohne die wichtigsten Grundlagen des Systems anzugreifen. Eine Analyse des ,Entwicklungsmodells" von 1971 - 1975 zeigt, daß die ,populistischen“ Ziele des Blocks an der Macht in der ersten Hälfte des Fünfjahresplans nur noch dadurch erreicht werden konnten, daß alle verfügbaren politischen und ökonomischen Reserven des Landes sowie die Auslandshilfe in die Waagschale geworfen wurden. Dieser „Populismus“ wurde jedoch in der zweiten Hälfte des Fünfjahresplans, als das Investitionsprogramm ensetzte, zum Hemmschuh.

Obwohl die offizielle Propaganda immer noch ihre Mythologie von, großen Investitionen und hohen Löhnen" kultivierte, war in Wirklichkeit schon die Walze der blitzartig wachsenden Akkumulationsrate ins Rollen gekommen. Diese Rate belief sich für 1974 und 1975 auf $38 \%$ bzw. $37,8 \%$ und brach alle bekannten Rekorde (inklusive Japans und Brasiliens) (4). Die Beibehaltung der hohen Reallohnzuwachsrate wurde unmöglich, gleichzeitig aber auch die Rücknahme der Propaganda von den ,,hohen Löhnen“. Und so mußte auf die Welle der Akkumulation eine peinlich versteckte Inflationswelle folgen. Da das „Statistische Jahrbuch“ für Polen keine Inflation kennt, müssen wir die Angaben über die Geldzirkulation untersuchen. Sie belief sich 1970 auf 58,64 Mrd. Zloty, 1971 - 67,32 Mrd, 1972 - 78,19 Mrd.Zloty. Für 1973 wuchs die Rate auf 91,31 Mrd. Zl., dann 1974 auf 117,15 Mrd. Z1., und schließlich 1975 - 141,19 Mrd. Zloty (5). Wir müssen noch einmal erwähnen, daß die Fluktuation von 34 Mrd. Zloty im Jahre 1975 nur auf die extrem hohe Akkumulationsrate zurückzuführen ist, wobei gleichzeitig der Anteil der landwirtschaftlichen Investitionen an dieser Akkumulation (das Problem der Versorgung des Konsumgütermarkts) in den Jahren 1971 - 1975 abnahm (6).

Alle Versuche, einen Kompromiß zu finden zwischen der hohen Akkumulationsrate, der wachsenden Verschuldung auf den Westmärkten (wenigstens 9 Mrd \$ für Anleihen und Kredite und 6,5 Mrd \$ infolge der angehäuften Schulden in der Handelsbilanz 1971 - 1975 (7) und der zunehmenden Geldmenge in den Händen der

4 Rocznik Statysticzny (Stat. Jahrbuch) 1976, Warschau 1976, XXXIV - XXXV, Tab. 1.

5 Ebd., S. 504, Tab. 788.

6 Ebd., S. 125, Tab. 180 (meine eigenen Berechnungen). 1971 beliefen sich die (staatlichen, genossenschaftlichen und privaten) Investitionen auf $15,4 \%$ des gesamten Investitionsfonds, 1972-14,4\%, 1973-13,5\%,1974-13,1\% und 1975-13,2\% (in laufenden Preisen).

1976, S. 608, Tab die gesamte negative Handeisbilanz nach dem Statistischen Jahrbuch tember 1976. Tab. 979. Die Summen von Anleihen und Krediten nach „,Time" vom 6.Sephaben mehrere "Time" schrieb: „Zur Bedienung von anleihen-suchenden Kommunisten haben mehrere amerikanische Banken in Osteuropa Filialen eröffnet. (. . .) Das Geschäft 
Bevölkerung, wurden allein durch das Prinzip des ,neuen Modells" selbst vereitelt, das vor allem in der Beibehaltung der grundlegenden Charakteristika des ökonomischen und sozialen Systems bestand, welches auf zentralistisch-bürokratischem Management und Planung sowie auf dem gesellschaftlichen Interessenvertretungsmonopol der bürokratisch-technokratischen Schichten basiert (8). Wo die ansteigende individuelle Produktionsleistung einen bedeutenden Produktionszuwachs ermöglichte und wo die Arbeiter im Vertrauen zum Block an der Macht zusätzliche Produktion in Angriff nahmen, da führte gleichzeitig das sozio-ökonomische System zu einer Zerstörung der individuellen Anstrengungen.

Man brauchte die Ánstrengungen der gesamten Gesellschaft, um die Produktion um $20 \mathrm{Mrd}$. Zloty zu erweitern; die Entscheidung einer Gruppe war aber ausreichend, um in der landwirtschaftlichen Produktion schlagartig eine Krise auszulösen und mehrere Milliarden Zloty zu verschwenden (9). Dasselbe in der Industrie. Der Fetisch der individuellen Produktivität, der Fetisch von „Müßiggängern“ usw. diente als allerbeste Entschuldigung für die makro-ökonomische Verschwendung, zur Maskierung der Grundlagen des bürokratischen ,Wirtschaftswunders“.

Ein bürokratisches, ,Wunder ${ }^{66}$ kann jedoch Erfolge immer nur auf dem Papier erzielen, nicht aber die Läden mit Fleisch, Butter und Eiern versorgen. Auf der Suche nach gesellschaftlicher Unterstützung initiierte die Gruppe an der Macht relativ schnell die Produktion des „Fiat“, mittlerer elektronischer Geräte usw. Diejenigen, die diese Güter nachfragen, sind aber nicht diejenigen, die den größten Teil des Volkseinkommens produzieren. Und für die sind vor allem „Basisgüter" - Nahrungsmittel, billige Stoffe, bequeme Massenverkehrsmittel und Kommunikationsmittel, Wohnungen und billige Möbel, gleiche Ausbildungschancen für die Kinder, auch auf Universitätsebene, und damit der Bau von Wohnheimen in den Wissenschaftszentren, ein allgemeines Gesundheitssystem, preiswerte Ferien usw. - wichtig. Die Befriedigung dieser Ansprüche erfordert aber beschleunigte Produktion solcher „Basis"-Güter wie Zement, Baumaterialien, maschinelle Hilfsmittel für die Bauern usw. Aber diese Güter fehlen noch, und ihre Produktion wächst erheblich langsamer als die der "Elite". Artikel.

Als im Juni 1976 die Sicherheitsgrenze des Systems erreicht wurde und die (im wörtlichen Sinne) bankrot te Regierung Zuflucht nahmzu ihrem letzten Argument, einer drastischen Preiserhöhung, verbunden mit einem prozentualen Lohnausgleich (nach dem Motto: wer schon hat, dem wird noch mehr gegeben), da wurde plötzlich der enge Zusammenhang zwischen makro-ökonomischer Verschwendung, Fehlern in der Politik und langfristigen Veränderungen in der Struktur von Nachfrage und Produktion ersichtlich. Genauso deutlich kam die gesellschaftliche Basis des Blocks

hat sich gelohnt. Kommissionen und andere Gebühren können sich auf bis zu 2 Millionen $\$$ belaufen für eine 200 Mio $\$$ Anleihe - und das umfaßt nicht einmal die späteren Zinsgebühren." (S. 41).

8 Zum gegenwärtigen Zeitpunkt findet sich die beste Analyse in: Wlodzimierz Brus, Ogolne problemy funkcjonowania gospodarki socjalisycznej (Allgemeine Probleme der Funktionsweise der sozialistischen Ökonomie), Warschau 1961.

9 Einige Angaben wurden in der Parlamentsrede gemacht, die Premierminister Piotr Jaroszewicz bereits nach Niederschlagung der Streiks gehalten hat. 
an der Macht ans Licht, die größten Konsumenten des Systems, die bereit sind, eine Erhöhung der Fleischpreise um ein paar Zloty hinzunehmen, wenn sie nur ihren "Fiat" behalten können und ihre importierten Konserven usw. Es wurde auch deutlich, wer der gefährlichste Gegner des Systems war --die Arbeiterklasse und die Bauernschaft.

\section{B. Charakteristischer Zeitpunkt der ökonom ischen Krise}

Dieser Gegner trat nun schon zum zweitenmal in den letzten 6 Jahren an die Öffentlichkeit, aber unter ganz anderen Umständen. Trotz der Heldenhaftigkeit der Werftarbeiter in Gdansk, Gdyngen und Szczecin wurde ihr Aufstand von 1970 vom Block an der Macht mit traditionellen Mitteln zerschlagen - durch Absetzung der Führung und das Erwecken von Hoffnungen. Und als die Welle der Unruhe voruber war, wurde alles wieder wie vorher. Dennoch war der Keim der jetzigen Ereignisse und der jetzigen Krise in dieser ,Tradition" schon angelegt.

Aufgrund der bürokratischen Illusionen, die der Gesellschaft vorgegaukelt wurden und nicht selten die Gruppe an der Macht selbst irreführte, trafen sich der konservative Radikalismus der Wirtschaftspolitik und der Radikalismus, der zur Verteidigung des alten Systems alle erreichbaren sozialen und ökonomischen Reserven aufs Spiel setzte und so zu den negativen Aspekten des Systems führte, in einer konkreten Entscheidung am 24. Juni 1976. Dieser Radikalismus setzte die soziale Unzufriedenheit frei und beraubte das System gleichzeitig aller inneren Reserven, die eine „populistische" Haltung hätten ermöglichen können, wie es schon im Februar 1971 - nach dem entscheidenden Streik der Textilarbeiterinnen in Lodz - der Fall gewesen war. Heute kann nur ,brüderliche" Hilfe aus Ost und West die Rückkehr zu einem instabilen, kurzlebigen Gleichgewicht ermöglichen. In dem Bemühen um die Erhaltung des status quo bleibt ihnen keine andere Wahl (10) (Zur Zeit wird diese Hilfe reichlich, gemeinsam und mannigfach gegeben.) Der Block an der Macht hat derzeit keinerlei innere Ressourcen zur Verfügung. Und dies ist einer dër Haüptğrunde dafür, daß wir ein erstaunliches, tragisches und gleichzeitig komisches Phönomen beobachten können: Polen hat keine Regierung, die heute bereits ein ökonomisches Programm zur Verbesserung der Lage präsentieren könnte.

\section{Die Reaktion des Blocks an der Machet}

Die Arbeiterunruhen vom Juni 1976 in Ursus und Radom entstanden vor allem aus tiefster Verzweiflung. Die Leute waren wütend. Sicher, in den vorhergehenden 6 Jah-

10 Symptomatisch dafür war die Reaktion der westlichen bürgerlich-liberalen Presse auf die Ereignisse in Polen

Trotz einer gewissen Schadenfreude dominierte einfach die herzliche Hoffnung, ,die Regierung werde es schon irgendwie schaffen". Vgl.z. B.P. Osnos, Poland: Balm for Angry Public, ,, The Guardian Weekly", 7. November 1976. 
ren hatte es beträchtliche Fortschritte auf vielen Gebieten gegeben, aber sie kamen nicht allen sozialen Gruppen und Klassen gleichmäßig zugute. Darüber hinaus wurden die Grundbedürfnisse nicht ausreichend befriedigt, was alle Statistiken gleich ausweisen. Im Juni 1976 beschloß die Regierung, die im Dezember 1970 die „Revoluion der Hoffnung" proklamiert hatte, den Lebensstandard des größten Teils der Bevölkerung zu senken, und das in einem Ausmaß, das nur mit den Beschlüssen von 1953 (beschleunigte Industrialisierung) oder mit Stalins Fünfjahresplan von 1930 vergleichbar ist, da zu den von der Gomulka-Gruppe im Dezember 1970 projektierten Preiserhöhungen noch der Beitrag der jetzigen Führungsgruppe von 1971 - 1975 hinzukam. Während Gomulka demnach einen Preisanstieg um 30 - 35\% gefordert hatte, heißt es jetzt, daß zur Finanzierung von Krise und Verschwendung eine 60 65 \%ige Steigerung notwendig sei. Aus diesem Grund haben wir statt einer , Revolution der Hoffnung“ eine „Revolution der Hoffnungslosigkeit“.

Der Block an der Macht reagierte auf die Verzweiflung der Arbeiter mit gleicher Unsicherheit: Innerhalb von 24 Stunden wurde die Entscheidung zurückgenommen; gleichzeitig wurden Versuche gemacht, eine Unterstützung für sie zu organisieren und die Arbeiter mit Hilfe der Polizei und des Sicherheitsdienstes zu terrorisieren. Hunderte yon Arbeitern wurden verhaftet, und die ersten Gerichtsurteile waren wirklich grausam: Gefängnis zwischen 2 und 10 Jahren. In den vom Streik betroffenen Unternehmen führte das Management mit Unterstützung von Partei und Gewerkschaftsfunktionären eine Säuberung unter den Arbeitern durch; Hunderte von FamiIen waren plötzlich ohne Lebensunterhalt.

Diese Terror welle wurde aber verhältnismäßig schnell gedämpft. Das überrascht nicht, daß außer dem Nahziel, die Ausbreitung der Streiks um jeden Preis zu verhindern, der Terror in der derzeitigen Situation keine konstruktive ökonomische Funktion haben kann. Obgleich der Terror die Krisenerscheinungen eingedämmt und zeitweise im Augenblick eine Umsetzung in eine gesamtgesellschaftliche Bewegung verhindert hat, welche nicht nur ökonomische, sondern auch politische Forderungen vertreten hätte, so hat er doch nicht eine Lösung der ökonomischen Krise befordert. Weil er der Arbeiterklasse kein wirksames Programm zur Verbesserung der ökonomischen Situation „anbieten" konnte (das resultiert aus dem Fehlen jeglicher echter Reserven), konnte der Terror nur hemmende Funktion haben - und das hauptsächlich auf der politischen Ebene.

Dieser begrenzte Charakter des Terrors nach dem 25. Juni 1976 wird nicht nur in der Politik des Blocks an der Macht gegenüber dem Sektor der Arbeiterklasse reflektiert (Ende August und vor allem im September und Oktober wurden die Aktivitäten der Unterdrückungsmaschinerie erheblich gebremst, und eine Anzahl von Gerichtsurteilen wurden noch einmal ,überprüft“, allerdings ohne Zurücknahme der formalen Anklage eines „Verbrechens"; ein Teil der verurteilten Ursus-Arbeiter wurde aus dem Gefängnis entlassen), sondern zeigt sich auch in der Politik gegenüber den Bauern und der städtischen Intelligenz.

Seit mindestens $21 / 2$ Jahren findet sich eine stetige Abnahme der landwirtschaftlichen Produktionsleistung. Das ist einerseits eine Folge der Politik des Blocks an der Macht, die zu einer verminderten Rentabilität vor allem in der Tierzucht führ- 
te (für die Bauern ungünstige Preispolitik bei landwirtschaftlichen und Industriegütern), und andererseits - unter dem Einfluß dieser Politik - einer Einschränkung der Investitions- und Produktionstätigkeit der Einzelbauern. Berücksichtigt man den verlänger ten Produktionszyklus in der Landwirtschaft, so zeigten sich die Auswirkungen der für die Bauern unvorteilhaften Politik in vollem Ausmaß erst 1975 und vor allem 1976 (11). Der Block an der Macht muß die Lösung der Situation allein auf der ökonomischen Ebene suchen, denn jeder Versuch des Terrors gegenüber den Bauern würde eine sofortige Verschärfung der Krise in der Landwirtschaft bedeuten und gleichzeitig die Notwendigkeit schaffen, das gesamte System nach den Maßstäben einer „Kriegswirtschaft" radikal zu reorganisieren. Berücksichtigt man alle inneren und äußeren Faktoren, von denen der Block an der Macht abhängig ist, dann verbietet sich ene solche Vorgehensweise von selbst. Das ist der Grund, warum der Block an der Macht zur Lösung der Krise in der Landwirtschaft seine Wirtschaftspolitik gegenüber der individuellen Landwirtschaft verändern muß, günstigere Tauschbedingungen schaffen, die Versorgung mit in der Landwirtschaft benötigten Industriegütern verbessern, die Preise für landwirtschaftliche Erzeugnisse anheben muß usw. Diese Landwirtschaftspolitik muß also in der Lage sein, die Unzufriedenheit der Bauern politisch $z u$ neutralisieren. (Und das geschieht bereits.) Und das bedeutet Liberalisierung, „Wohlwollen“, „Fürsorge“, und nicht Terror.

In bezug auf die städtische Intelligenz ist die Politik des Blocks an der Macht ähnlich, wenn auch von anderen Faktoren bestimmt. Da diese Schicht insgesamt keine Gefahr für die ökonomischen Grundlagen des Funktionierens des Systems darstellt und außerdem ein beträch tlicher Anteil auf die eine oder andere Art organisatorische Hauptstützen des Blocks an der Macht bildet oder die Wirtschaftspolitik von 1971 1975 befürwortet, ist es die einzige Sorge des Blocks an der Macht, oppositionelle Güppchen der Intelligenz von der Arbeiterklasse fernzuhalten. Zur Erreichung dieses Ziels ist keine große Änderung in der Politik des Blocks an der Macht nötig, da aus ihrer Sicht die Gefahr einer Allianz von oppositioneller Intelligenz und der Arbeiterklasse in der gegenwärtigen Situation relativ gering erscheint (12).

Auf der anderen Seite ist die eigenartige Empfindlichkeit des Blocks an der Macht gegenüber den Reaktionen der sog. öffentlichen Meinung, insbesondere in den westeuropäischen Ländern ein Element, welches die Politik des Massenterrors beträchtlich einschränkt. Diese Empfindlichkeit hat sicherlich weniger mit der Absicht zı tun, die Beschlüsse der Schlußakte der Konferenz von Helsinki zu verwirklichen, als mit der ökonomischen Situation im Inland. Da berücksichtigt werden muß, daß der Block an der Macht bis zu einem gewissen Grad vom Wohlwollen der Gläubiger im Westen abhängig ist und über $60 \%$ des polnischen Handels auf kapitalistischen

11 Nur aufgrund der Auslassung dieses wichtigen Aspekts des landwirtschaftlichen Produktionszyklus in der Propaganda war es möglich, die Krise in der landwirtschaftlichen Produktionsleistung als aileinige Folge der schlechten klimatischen Bedingungen hinzustellen. Vgl. hierzu den Aufșatz von H. Szlajfer, a.a.O.

12 Aufgrund der Erfahrungen vollständiger Verwirrung innerhalb der linken Opposition in der Intelligenz im Jahre 1968 und der Gleichgültigkeit der Intelligenz während der Aufstände von 1970 ist eine solche Haltung teilweise berechtigt. 
Märkten stattfindet, könnte nur äußerste Bedrohung den Block an der Macht veranlassen, wieder nach den alten Spielregeln zu verfahren: physische Unterdrückung, Gerichtsurteile, kurz - Massenterror gegen alle Gegner. Man sollte aber den Druck der progressiven Kräfte in den westlichen Ländern nicht unterschätzen, weil dieser Druck Stimulanzfunktionen hat für den Prozeß der Einschränkung der Bestrebungen gewisser Konzerne, mit Osteuropa zusammenzuarbeiten ohne Rücksicht auf die Politik (13). Im Falle der UdSSR hat dieser Druck vielfach schwerwiegende Erfolge erzielt; umso mehr Grund hat Polen, ihn zu berücksichtigen, weil Polen sehr viel stärker von der Konjunktur der kapitalistischen Wirtschaft abhängig ist. Natürlich wächst die „Empfindlichkeit" des Systems gegen Kritik von außen 1) mit der damit verbundenen wirtschaftlichen Drohung und 2) mit dem progressiven Charakter der Bewegung, die jeweils Kritik übt. Wenn z. B. Franz Josef Strauss meldet, daß in Polen die Grundrechte der Arbeiterklasse verletzt werden, hat das für den Block an der Macht keine Bedeutung, da dies ja nichts anderes bedeutet, als daß F. J. Strauss ein unermüdlicher Gegner des Sozialismus ist und daher von diesem Gegner nur Kritik erwartet werden kann. Wenn aber Enrico Berlinguer (KPI), Santiago Carillo (KPSp) oder Georges Marchais (KPF) so etwas verbreiten, dann muß der Block an der Macht wohl oder übel diese Kritik schlucken, und wenn zusätzlich die Krisensituation ungelöst ist, muß er auch noch guten Willen zeigen (14).

13 Vgl. A. Sampson, The Sovereign State of ITT, Greenwich, Conn. 1974, S. 294 - 295; P.L. Berger, The Greening of American Foreign Policy, ,Commentary“, 1976, Vol. 61, Nr. 3, S. 26. Niemand hat jedoch besser die Einstellung der großen Konzerne gegenüber Beziehungen mit den osteuropäischen Ländern gekennzeichnet als einer der reaktionärsten Gewerkschaftsführer, der Chef der AFL-CIO, G. Meany. Er sagte: ,Die amerikanischen Kapitalisten waren nur solange wütende Antikommunisten, wie sie ihre Geschäftsinteressen bedroht sagen - nicht weil sie um Demokratie und Freiheit besorgt waren. (. . .) Jetzt, wo sie sehen, daß im Handel mit den Kommunisten ein paar Dollars zu verdienen sind, haben sie ihre Haltung geändert. "Zit. nach "Commentary", 1976, Vol. 61, Nr. 6, S.4.

Die Einstellung der konservativen Kritiker zeigt sich genau in den Thesen von Norman Podhoretz, die wie folgt zusammengefaßt werden können: Die ökonomische Macht wird frïher oder später von dem Bewußtsein dieser Macht begleitet. Podhoretz schreibt: ,in der Tat haben unsere phantastische landwirtschaftliche Produktivität und die ständig technologische Erfindergabe unsere Macht gegenüber der der Sowjetunion vergrößert; und aufgrund der sowjetischen Abhängigkeit von unserem Getreide und unserer Technologie ist unser Einfluß auf die Sowjets jetzt so groß wie nie. Wenn wir diese Macht dazu benutzen wollen, ihnen $z u$ begegnen, sind wir durchaus in der Lage, eine Vielzahl von Maßnahmen zu ergreifen ..." (N. Podhoretz, Making the World Safe for Communism, ,Commentary", 1976, Vol. 61, Nr. 4, S. 41). Die fortschrittlichen Kräfte, insbesondere die kommunistischen Massenparteien in Italien, Frankreich und Spanien, können logischerweise keine ökonomische Macht ausüben. Dennoch ist der von ihnen ausgehende Druck nicht weniger wichtig. Im Gegenteil, die Stimme dieser Parteien hat ein entschieden größeres Gewicht als alle ökonomische Erpressung durch Regierungen und Konzerne. Darüberhinaus können nur diese Parteien, und ganz allgemein die Bewegung der Arbeiterklasse in den Hauptzentren der kapitalistischen Welt, auf lange Sicht zu Verbündeten der polnischen Arbeiterklasse und der demokratischen Freiheiten in unserem Lande werden. Dieser Prozeß nahm seinen Anfang in dem Augenblick, in dem das Interesse an einer autonomen Entwicklung der Arbeiterbewegung im Westen das direkte und grundlegende Ziel dieser Parteien wurde. Das beseitigt zwar nicht die Möglichkeit einer opportunistischen 
Eine Analyse der Reaktionen des Blocks an der Macht auf die Arbeiterstreiks zeigt also insgesamt: das Fehlen jeglicher innerer Reserven, die tiefe Vertrauenskrise innerhalb der Gruppe, die 1970 die ,,andauernde“ Verwirklichung der Forderungen der Arbeiter versprach, die Notwendigkeit, Zeit zu gewinnen, um Hilfe von auBen erhalten zu können (aus Ost und West); all das bewirkte in relativ kurzer Zeit einen stürmischen Umschwung in der Politik des Blocks an der Macht: von direkter Unterwerfung der streikenden Arbeiter hin zu einer Politik schrittweiser Zugeständnisse und kurzlebigem Waffenstillstand. Gleichzeitig reagiert der Block an der Macht sehr viel elastischer in der Erkenntnis, daß die wichtigsten Symptome der ökonomischen Krise noch nicht beseitigt sind, daß sich das ,Krisensyndrom" noch nicht aufgelöst hat und daß ein neuer Ausbruch der Arbeiterproteste droht. Er versucht, sich nicht noch andere gesellschaftliche Gruppen zum Feind zu machen und sie davon abzuhalten, sich der Protestwelle der Arbeiter anzuschließen. Das erklärt nicht nur die kurze Dauer der brutalen Unterdrückung, sondern auch ihre Beschränkungen und die Tatsache, daß der Terror nicht auf die Bauern bzw. die städtische Intelligenz ausgedehnt wurde.

\section{Charakteristische Züge des Arbeiterprotests}

Vor einer Diskussion möglicher Krisenlösungsmechanismen scheint es sinnvoll, einige charakteristische Züge der Protestbewegung der polnischen Arbeiter hervorzuheben, die sich sowohl im Dezember 1970 - Februar 1971 als auch im Juni 1976 zeigten. Dieser Exkurs soll ein Verständnis der spezifischen Formen, in denen in Polen eine unabhängige Bewegung der Arbeiterklasse entsteht, ermöglichen.

Im Gegensatz zu den Ereignissen von 1956, die in Polen mit „Polnischer Oktober" bezeichnet werden, waren die Massenstreiks der Arbeiter von 1970 und 1976 Ausdruck einer absolut autonomen Bewegung der Arbeiter der Großindustrie. Wenn die Arbeiterbewegung 1956 eine gewisse Grundlage hatte in Form der alten Vorkriegsund frühen Nachkriegstradition der unabhängigen Arbeiterbewegung und in der zeitweiligen Unterstützung des sog. anti-stalinistischen Flügels in der Partei (deren hervorragender Vertreter Wladyslaw Gomulka war), so umfaßten die Streiks 1970 und 1976 eine Arbeiterklasse, die ihrer Tradition und aktiver politischer Verbündeter beraubt war.

Um den Vergleich fortzuführen: Wenn die Arbeiterbewegung gleichzeitig 1956, unterstützt von Gruppen der sozialistischen Intelligenz und von einer inneren Zerstrittenheit des Blocks an der Macht, sehr schnell die geeigneten Formen ökonomischer und politischer Aktion fanden (Arbeiterräte), so erlangte die Bewegung von 1970 - 1976, wenn sie auch weitgehend keinen rein politischen Charakter hatte und

Haltung dieser Parteien gegenüber der Frage der Arbeiterbewegung und der demokratischen Freiheiten im Osten, aber die größten organisatorischen, ideologischen und politischen Hindernisse sind schon beseitigt. Die Berliner Konferenz der europäischen kommunistischen und Arbeiterparteien von 1976 ist ein wichtiges Anzeichen dafür, ebenso wie die Reaktionen der KP Italiens auf die Ereignisse vom Juni 1976 in Polen. 
unfähig war, gleich verläßliche Anführer und Vertreter zu bestimmen, doch einen ausgesprochenen Massencharakter und relative Unabhängigkeit.

Wie der Verlauf der Ereignisse nach 1956 zeigt, wurde der Faktor, der in der Zeit von Juni bis Oktober 1956 eine der Hauptstärken der Arbeiterbewegung war die Unterstützung des anti-stalinistischen Flügels -, auch zur Ursache ihrer Niederlage, sowohl politisch als auch ökonomisch. Die von diesem Flügel erlangte politische Führungsposition erlaubte es ihnen, nur die krassesten Wesenszüge des Stalinismus zu reformieren, seine Grundlagen jedoch beizubehalten. Zunehmend wurde auch die Selbständigkeit der Arbeiterbewegung abgeschafft, und das neue Gesetz über Arbeiterräte von Gomulk ka beendete die Stufe der unabhängigen Arbeiterpolitik gegen Ende der 50er Jahre. Die Arbeiterräte verloren alle wichtigen Befugnisse an die staatliche Verwaltung und die Partei- und Gewerkschaftsmaschinerie (15).

Die Arbeiterunruhen von 1970 in den Küstengebieten erwiesen sich als die ersten selbständigen und massenhaften Anzeichen der Entstehung einer neuen Arbeiterklasse, deren einzige Tradition die ihres eigenen Kampfes ist, den sie im Rahmen des gegenwärtigen sozio-ökonomischen Systems führt. Aus zwei Gründen handelt es sich um eine neue Arbeiterklasse:

Erstens sind die Mitglieder dieser Klasse im jetzigen System aufgewachsen und haben hier ihre erste Arbeit bekommen, sind also nicht ,belastet" mit der Notwendigkeit, ihre jetzige sozio-ökonomische Stellung mit den kapitalistischen Vorkriegsbedingungen vergleichen zu müssen. In den Jahren 1958 - 1968 nahm die Zahl der Industriearbeiter von 2,4 Mio bis auf 3,1 Mio zu und erreichte 19754 Mio (16). Wenn man das berücksichtigt und dazu die Tatsache eines dauernden "Generationswechsels", dann kann man mit Recht annehmen, daß der größte Teil der Arbeiter von 1971 - 1975 der „Volks-Polen Generation“ angehörte.

Zweitens richtet sich der Kampf dieser neuen Arbeiterklasse gegen den Block an der Macht, der sich auf das Staatseigentum an den Produktionsmitteln gründet und - auf der ideologischen Ebene - sozialistische Ziele vertritt. Das Neue an dieser Arbeiterklasse besteht also darin, daß sie die Legitimation dieser Gruppe infrage stellt, ihr Verbündeter und gleichzeitig Repräsentant zu sein - ohne dabei den $M y$ thos von freiem Unternehmertum und kapitalistischem Wettbewerb zu wiederholen, Alle Einschätzungen der Ereignisse von 1970 und 1976 weisen deutlich darauf hin, daß die Arbeiterklasse die Hegemonie des Blocks an der Macht nicht von einer ,,konkurrierenden" ideologischen Position her in Frage stellt, sondern sich auf die Notwendigkeit praktischer Verwirklichung der von den Ideologen (des Blocks an der Macht) gepredigten Forderungen beruft. In diesem Zusammenhang scheint es auch unbezweifelbar, daß trotz der derzeit kursierenden Interpretationen der Ereignisse in Polen als auf sozialdemokratische Zielvorstellungen und Formen einer sozialen

15 Vgl. J. Balacerek und L. Gilejko, Roswoj samorzadu robotniczego (Die Entwicklung der Arbeiterselbstverwaltung), in: Polityka gospodarcza Polski Ludowej (Die Wirtschaftspolitik Volkspolens), Bd. II, Warschau 1962,

16 L. Beskid und K. Zagorski, Robotnicy na tle przemian struktury spolecznej w Polsce (Die Arbeiter im Zusammenhang der Veränderungen der Sozialstruktur Polens), Warschau 1971, S. 70, Tab. 44; Rocznik Statystyczny 1976, S. 182, Tab. 248 und S. 184, Tab. 250. 
Marktwirtschaft orientiert (17), die nene Arbeiterklasse in idealer Weise sozialistische Tendenzen und Ziele repräsentiert. Die Verwirklichung dieser Ziele ist jedoch nur möglich unter Bedingungen einer Arbeiterdemokratie, die auf der wirtschaftlichen Macht von Arbeiterräten und Parlament basiert und direkt von den Arbeitern der Großindustrie unterstiitzt wird. Eine solche Demokratie kann aber - und das muß betont werden - nur verwirklicht werden unter Weitergabe dieser demokratischen Freiheiten an die Gesamtgesellschaft, d. h. nach vorheriger Brechung des politischen Monopols des Blocks an der Macht (18).

Im jetzigen Entwicklungsstadium jedoch geht die Bewegung der Arbeiterklasse nicht über die - in der Regel negative - Formulierung momentaner ökonomischer Forderungen hinaus. Auf der anderen Seite jedoch ist diese niedrige ,theoretische Ebene" der formulierten Forderungen begleitet von viel besser entwickelten Organisationsformen dieser Bewegung, wenn sie in direkte und offene Auseinandersetzungen mit dem Block an der Macht eintritt. Gemeint ist hier die spontante Gründung von Streikkomitees, die Übernahme von Verwaltungsfunktionen in den streikenden Betrieben, usw. Dieses Phänomen kann aber nicht überraschen, wenn man die technische Struktur der modernenindustriellen Produktion und damit zusammenhängend den Koeffizient der Konzentration der Arbeiterklasse in großen Industriebetrieben berücksich tigt.

Nach der Statistik gab es 1975 in Polen 18.900 (staatliche) Industriebetriebe mit etwa 5 Millionen Beschäftigten (19). Die Betriebe mit über 2000 Beschäftigten machten $2 \%$ aller Betriebe aus (ca. 380), und sie beschäftigten etwa 2 Millionen

17 Eine solche Interpretation wird tatsächlich von Jacek Kuron vertreten.

18 Im Zusammenhang der Erfahrungen der UdSSR, Polens und anderer osteuropäischer Länder sind die Bemerkungen Rosa Luxemburgs von 1918 wahrlich prophetisch. Sie schrieb: „,Der Grundfehler Lenin-Trotzkischer Theorie ist eben der, daß sie die Diktatur, genau wie Kautsky, der Demokratie entgegenstellen. ,Diktatur oder Demokratie' heißt die Fragestellung sowohl bei den Bolschewiki wie bei Kautsky, Dieser entscheidet sich natürlich für die Demokratie, und zwar für die bürgerliche Demokratie, da er sie eben als die Alternative der sozialistischen Umwälzung hinstellt. Lenin-Trotzki entscheiden sich umgekehrt für die Diktatur im Gegensatz zur Demokratie und damit für die Diktatur einer Handvoll Personen, d. h, für bürgerliche Diktatur.Es sind zwei Gegenpole, beide gleichweit entfernt von der wirklichen sozialistischen Politik. Das Proletariat kann, wenn es die Macht ergreif $t$, nimmermehr nach dem guten Rat Kautskys unter dem Vorwand der ,Unreife des Landes“ auf die sozialistische Umwälzung verzichten und sich nur der Demokratie widmen, ohne an sich selbst, an der Internationale, an der Revolution Verrat zu üben. Es soll und muß eben sofort sozialistische Maßnahmen in energischster, unnachgiebigster, rücksichtslosester Weise in Angriff nehmen, also Diktatur ausüben, aber Diktatur der Klasse, nicht einer Partei oder einer Clique, Diktatur der Klasse, d. h. in breitester Öffentlichkeit, un ter tätigster ungehemmter Teilnahme der Volksmassen, in unbeschränkter Demokratie. " (S. 362 363) Und als logische zwingende Schlußfolgerung: „F reiheit nur für die Anhänger der Regierung, nur für Mitglieder einer Partei - mögen sie noch so zahlreich sein - ist keine Freiheit. I'reiheit ist immer Freiheit der Andersdenkenden. Nicht wegen Fanatismus der,Gerechtigkeit ${ }^{6}$, sondern weil all das Belebende, Heilsame und Reinigende der politischen Freiheit an diesem Wesen hängt und seine Wirkung versagt, wenn die ,Freiheit ${ }^{6}$ zum Privilegium wird." (S. 359, Fußnote 3). Rosa Luxemburg, Zur Russischen Revolution, in: Gesammelte Werke, Bd. 4, Berlin (DDR) 1974. 
Leute, also etwa $40 \%$ aller in der Industrie Beschäftigten. Wenn wir diese Häufung noch erweitern durch die Zahl der Betriebe mit über 1000 Beschäftigten, dann gab es 1975 etwa 850 von ihnen mit zusammen 2,5 Millionen Arbeitern, d. h. $57 \%$ aller Industriearbeiter (20). Industrien mit einer hohen Konzentration von Beschäftigung und Produktion sind: Brennstoffe/Energie, Metall-, Chemie- und elektrotechnische Industrie. Dagegen haben die Leicht- und Lebensmittelindustrie sehr niedrige Konzentrationskoeffizienten.

Während der Dezember 1970 - Februar 1971- und Juni 1976-Streiks traten organisierte Aktionsformen - in Form von Streik-Komitees - fast ausschließlich in großen Industriebetrieben auf, da in diesen Betrieben jede Artikulation von Widerstand gegen den Block an der Macht und die Formulierung einfachster Forderungen nicht möglich sind ohne ein Minimum an Organisation - ganz zu schweigen von der Notwendigkeit, diese Werke vor Zerstörung durch das unkontrollierte Anhalten technologischer Prozesse zu schützen (z. B. verursachte der Streik in den Petrochemischen Werken in Plock keinen materiellen Schaden; dasselbe gilt für die Werke in Ursus und Radom und die Streiks von 1970 in der.Reparaturwerft von Szczeczin, wo die streikende Belegschaft besondere Schutzmaßnahmen traf, um Schäden (beabsichtigte oder unbeabsichtigte) zu verhindern. Dagegen vollzog sich der große Streik der Textilarbeiterinnen in Lodz (Industrien mit sehr niedrigem Konzentrations-Koeffizient und breiter Streuung) nach dem herkömmlichen Mechanismus spontaner Streiks, ohne alle Elemente von Organisation und Führung.

An dieser Stelle erscheint aber ein überraschendes Paradoxon in der Entwicklung und Effektivität der Arbeiterbewegung in Polen. Wenn wir uns daran erinnern, daß sie noch auf der Stufe der Formulierung negativer Forderungen (,Runter mit den Preisen" usw.) ist, können wir die These formulieren: Die Effektivität des Widerstands der Arbeiter ist direkt proportional dem spontanen Charakter und dem Fehlen jeder Organisation und Führung. 1970 konnte die Staatsführung die perfekt organisierten Arbeiter der Szczecziner Werft von der Unmöglichkeit der Rücknahme der Preiserhöhung überzeugen. Den Anführern des Streiks fehlte die Erfahrung, ihre Hilflosigkeit gegenüber den Argumenten der neuen Staatsführung führte zum de facto Ertolg der neuen Staatstührung.

Einige Tage später brach in Lodz ein unkoordinierter Streik aus. Er erfaßte das gesamte Industriezentrum, aber gleichzeitig gab es niemanden, mit dem die Vertreter des Blocks an der Macht verhandeln konnten. Sie mußten aufgeben oder versuchen, die Streiks in Lodz mit Gewalt niederzuschlagen - und damit ein Wiederaufleben der Streikwelle im ganzen Land riskieren. Deshalb entschieden sie sich für Kapitulation.

Im Juni 1976 umfaßte die Streikbewegung den größten Teil der großen Industriebetriebe, aber nur in Ursus und Radom gab es Zusammenstöße der Streikenden mit der Polizei und den Sicherheitskräften. Diesmal riskierte der Block an der Macht keine Verhandlungen mit Streikkomitees, und, einmal den Ereignissen einen Schritt voraus, nahm er sofort seinen Beschluß zurück. Das führte fast automatisch zur Selbstauflösung der Streikkomitees.

$\mathrm{Da}$ der Block an der Macht sich jedoch nicht darauf beschränkte, seinen Be- 
schluß zurückzunehmen, sondern gleichzeitig repressive Maßnahmen ergriff, stand die Arbeiterklasse vor der neuen Aufgabe, nicht nur das direkte ökonomische Ziel zu erreichen, sondern auch ihre Genossen zu verteidigen, die gefangen gesetzt wurden bzw. ihre Arbeit verloren hatten. Eine solche Handlungsweise erfordert aber im Gegensatz zur bisherigen eine viel breitere Perspektive. In diesem Falle ist das Ziel nicht der unmittelbare ökonomische Gewinn, sondern ein „, nicht-meßbarer" Faktor - Solidarität, Verteidigung der Kollegen, und gleichzeitig Kampf für das Recht auf Versammlung, auf gemeinsamen Widerstand.

Die Lösung dieser Aufgabe erfordert ,permanente Aktion“, nicht eine augenblickliche Anstrengung. Die Organisation solcher Aktionen ist grundsätzlich nur im Rahmen des großen Industriebetriebes möglich, der den Massencharakter der Bewegung sichert und gleichzeitig wichtiges Element der gesamten Wirtschaft ist. So muß also die Spontaneität des Streiks abgelöst werden vom Zusammenhang der organisierten Bewegung. Der Brief von 899 Ursus-Arbeitern an die Behörden, der die Wiedereinstellung ihrer Arbeitskollegen forderte, belegt die Möglichkeit der Anpassung der bisherigen Widerstandsformen an die zu lösenden Aufgaben (21). Wir können auch in der Tat Anzeichen der inneren Entwicklung der unabhängigen Bewegung der Arbeiterklasse beobachten - unabhängig auch auf der ideologischen und politischen Ebene, daher nur im Vertrauen auf ihren eigenen Kampf. Ob sich diese Einschätzung bewahrheiten wird, ist weitgehend abhängig von der Entwicklungsrichtung der derzeitigen Situation und den Formen der Krisenbeseitigung.

\section{E. Liberalismus und die ökonomische Krise}

In seinem „Note sul Machiavelli“" stellte Antonio Gramsci die interessante Hypothese auf, daß im Gegensatz zu den entwickelten Systemen der Klassenherrschaft im Westen diese Systeme in den östlichen Gesellschaften ersetzt werden durch einen Despotismus des Staatsapparats. Diese Gegenüberstellung diente ihm als Ausgangspunkt für seine Analyse der notwendigen Modifikationen für eine Strategie revolutionärer Bewegungen und für seine Thesevon der Notwendigkeit, das ,Über raschungs“" moment durch hoch entwickelte Bündnisse und eine flexibel interpretierte Politik der Schaffung eines hegemonistischen Machtblocks zu ersetzen (22).

Unter den Bedingungen in Polen 1970 - 1976 trifft Gramscis Hypothese nur insoweit zu, als der Block an der Macht bis jetzt nicht in der Lage war, seine politische und ökonomische Hegemonie in eine ideologische und kulturelle zu verwandeln (im Falle der Bauern ist nicht einmal die ökonomische Hegemonie vollständig), seine 20 Ebd.

21 Auszüge aus diesem Brief lauten wie folgt: „Wir Arbeiter der Mechanik-Fabriken, Ursus fordern die Wiedereinstellung all derer, die wegen des Streiks und der Demonstration vom 25. Juni 1976 entlassen wurden. (. . .) Wir fordern, daß sie ihre vorherigen Arbeitsplätze wiederbekommen und damit alle Rechte, die mit der weiteren Beschäftigung einhergehen. Wir sind überzeugt, daß wir nur dann, zusammen mit allen Polen, die schwierige ökonomische Situation unseres Landes in dieser Zeit lösen können."

22 Vgl. Antonio Gramsci, Pisma Wybrane (Ausgewählte Werke), Warschau 1961, Bd. I, S. $585-587,611$. 
Herrschaft also keineswegs ein sicheres System von „Wällen und Gräben“ darstellt. Die Folge ist die spezifische Anfälligkeit des gesamten Systems für den Schock auch nur des kleinsten Widerstandes und Unzufriedenheit sowie die Notwendigkeit, in solchen Fällen, den ganzen Apparat der Unterđrückung mobilisieren zu müssen.

Es wäre aber falsch, wollte man behaupten, daß sich der Einflußbereich des Blocks an der Macht in den über 30 Jahren seiner Existenz nicht erweitert hätte. Das System hat $u$. a. den Kreis seiner Verbündeten und Nutznießer erweitert. Die dichotome Vorstellung von der „Diktatur einer Handvoll Bürokraten" ist heute genauso unsinnig wie das Gegenteil - das Konzept der ,klassenlosen Gesellschaft", wie es Chruschtschow am Anfang der 60er Jahre beschlossen hat. Wie wir schon betont haben, erzeugte die ökonomische Politik der Jahre 1971 - 1975 besonders günstige Voraussetzungen für die Stärkung des Blocks ökonomischer und sozialer Interessen, die nicht nur eine schmale Schicht der obersten Partei- und Staatsbürokratie betraf, sondern auch die „Arbeiteraristokratie“ und die oberen Schichten der städtischen Bevölkerung. Aus diesem Grund traf die Krise von 1976 zwar alle, aber verschiedene Schichten mit unterschiedlicher Gewalt. Und das bedeutet, daß der Block an der Macht nicht nur vor der Aufgabe steht, die ökonomische Krise im Rahmen ,,potentieller politischer Gefahr" zu halten, sondern auch in begrenztem Umfang eine gewisse Liberalisierung zu betreiben, insbesondere im Hinblick auf das intellektuelle Milieu des Landes.

Mitte September 1976 wurde in Warschau von einer Gruppe von Intellektuellen das Komitee zum Schutz der Arbeiter (KOR) gegründet. Sein Ziel ist finanzielle und juristische Hilfe für verfolgte Arbeiter. Die vom Komitee herausgegebenen „Komunikaty (,Communiques") informieren über Ausmaß und Formen der Unterdrückung und des Widerstands. Bis jetzt wurden die Aktivitäten des Komitees noch nicht durch Repressionen der Staatsmaschinerie behindert, und die Mitglieder und Sympathisanten des Komitees konnten mit ihrer Finanz- und Rechtshilfe eine Anzahl Arbeiterkreise erreichen, besonders in Ursus und Radom. Natürlich ist es schwer, vorherzusagen, wann der Block an der Macht gegen das Komitee vorgehen wird; im Augenblick jedoch ist es weder für den Block an der Macht ratsam noch bedrohen die Aktivitäten des Komitees das System direkt.

Das ändert jedoch nichts an der Tatsache, daß die Gründung des Komitees eine entscheidende Abkehr vom bis dahin "monolithischen" gesellschaftlich-politischen System darstellt, und die Kontakte, die das Komitee zu Kreisen der Arbeiterklasse aufgenommen hat, sind seit dem ,Polnischen Oktober" der erste Versuch, die Sackgasse zu überwinden, zu der die offizielle Politik der politischen Isolierung der einzelnen Schichten und Klassen voneinander geführt hat. Deshalb genießen die Aktivitäten des Komitees trotz ihres begrenzten Charakters und der von den Mitgliedern vertretenen ,Ideologien" breite gesellschaftliche Unterstützung, so daß man mit Recht sagen kann, daß die Praxis des Komitees den Ansichten der einzelnen Mitglieder weit voraus ist. Und in diesem Sinne werden die Grïndung und die Aktivitäten des Komitees dem Block an der Macht eine harte Nuß zu knacken geben.

Die passive Duldung der Aktivitäten des Komitees durch den Block an der Macht zeugt natürlich von einer gewissen Schwäche. Es zeigt auch die Grenzen auf, 
die möglicher Repression durch die Krise und die äußeren Verhältnisse gesetzt werden. Andererseits dürfen die Trümpfe des Blocks an der Macht nicht unterschätzt werden.

Die ökonomische Krise folgte einem jahrelangen extrem schnellen ökonomischen Wachstum, verbunden mit einem beträchlichen Reallohnanstieg in der ersten Hälfte des Fünfjahresplans. Obwohl die Hoffnungen und Illusionen der Arbeiterklasse im Juni 1976 brutal unterdrückt wurden, heißt das nicht, daß sie aufgegeben wurden. Es hat den Anschein, als hätte die Gruppe an der Macht trotz derzeitiger verbreiteter Resignation und Desillusion immer noch die ideologische und politische Kontrolle über die Arbeiterklasse, und das umso mehr, als - trotz der Gründung des Komitees zum Schutz der Arbeiter - bis jetzt noch kein rivalisierender ,ideologischer und politischer $\mathrm{Pol}^{\text {" }}$ entstanden ist, der im Sektor der Arbeiterklasse dem Block an der Macht ebenbürtig wäre.

So paradox es erscheint, so ist doch das Andauern der ökonomischen Krise - in gewissen Grenzen - für den Block an der Macht von Vorteil, da es in der derzeitigen politischen Konstellation das Bemühen um Restabilisierung fördert. Berücksichtigt man dazu, daß wir in absehbarer Zeit Auslandshilfe erwarten können, so heißt das, daß der Block an der Macht wenigstens zeitweilig Stabilität schaffen und die gröbsten Auswirkungen der ökonomischen Krise wahrscheinlich wird beseitigen können.

Wenn also in den kommenden Monaten nicht eine rapide Verschlechterung der Situation eintritt oder ein offener Konflikt in einem wichtigen Industriesektor (was nicht auszuschließen ist) oder innerhalb der herrschenden Gruppe ausbricht, erscheint ein Umschlagen der jetzigen Krise in eine offene politische Krise nicht wahrscheinlich. In Anbetracht der Schwere der gegenwärtigen Krise und der Tatsache, daß die Einführung bestimmter Änderungen in der Produktionsstruktur Zeit braucht, scheint der Block an der Macht jedoch nicht willens, weitergehtende repressive Maßnahmen gegen die städtische Intelligenz und vor allem gegen das Komitee zum Schutz der Arbeiter zu ergreifen, bevor ein einigermałsen stabiles Gleichgewicht erreicht ist.

Im Hinblick auf die Chancen für die Schaffung einer unabhängigen Arbeiterbewegung hat die derzeitige Krise uns die Notwendigkeit sehr viel breiterer und festerer Beziehungen zwischen der Arbeiterklasse und den Gruppen der städtischen Intelligenz vor Augen geführt. Beim gegenwärtigen Stand der Entwicklung dieser Bewegung muß dieser Kontakt sich natürlich auf „Aufklärungsaktionen“ beschränken. Diese würden einerseits Aufklärung der Arbeiter über das Ausmaß der ihnen zustehenden Rechte beinhalten und andererseits darin, sie mit den Traditionen der unabhängigen Arbeiterbewegung und bestimmten Kampfformen und ideologischen Traditionen bekannt zu machen. Besonders wich tig ist auch, Perspektiven für den aktuellen Widerstand zu zeigen, für die Möglichkeit des Kampfs am eigenen Arbeitsplatz bei den Wahlen zu den Betriebs- und Arbeiterräten (offiziell), bei der Verteilung der Produktionsaufgaben, bei der Leistung der sog. ,gesellschaftlichen Arbeiten“"usw. Das ist der einzige Weg, dauerhafte und reale Bedingungen fur Solidarität zu schatten, da das nur in gemeinsamer Aktion geschehen kann, durch praktische Erprobung und 
mit Rückschlägen und Fehlern. Und wenn unsere Einschätzung der gegenwärtigen Situation richtig ist, dann ist der Spielraum für solche autonomen Aktionen bereits geschaffen.

\section{DIE LINKE OPPOSITION IN DER SOWJETUNION sechs Binde 1923 - 1928}

Herausgegeben und eingekeitet von Uff Woller

Diese Anthologie dokumentiert anhand der Originaltexte die grundlegende Debatte um den Aufbau des Sozialismus in der SU. Damit wird es erstmalig einem breiten Publikum ermöglicht, sich selbst ein Bild vom Verlauf dieser zentralen Auseinandersetzungen zu machen und die Grund lage für das Verständnis der aktuellen Debatten um den Eu rokommunismus und die Dissidentenfrage geboten. Deutsche Erstveröffentlichungen von Lenin,Trotzki, Bucharin,Preobraschenskij, u.a.

(bei Best. des Gesamtwerks - ISBN 3921241081 - pro Band DM 1.- Ermäßigung)

Im Herbst erscheint Band VI, Texte von 1927-28 ca. 600 Seiten, ca. DM 29. ISBN 3921241197

Dieser Band dokumentiert mit dem 15.Parteitag das Ende der Fraktionskämpfe in der KPdSU und den Ausschluß der Opposition. Vor genau 50 Jahren waren damit die Weichen für die weitere Entwicklung der $S U$ gestellt.

Als Abschluß dieser Anthologie erscheint im Herbst ein Diskussionsband:

DIE SOZIALISMUSDEBATTE

Mit Beiträgen von E.Mandel, J.Pelikan, B.Rabehl, H.Fleischer, J.Moneta, P.Frank, R.V.Daniels, P.v.Oertzen, J.Steffen, O.K.Flechtheim, u.v.a.

Von durchaus unterschiedlichen wissenschaftlichen und politischen Ansätzen wird eine breite und kritische Dis kussion über Geschichte und Gegenwart des Sozialismus geführt, die bis zum Eurokommunismus und der Dissidentenfrage reicht.Die Palette der Autoren bürgt nicht nur für ein breites Meinungsspektrum,sondern auch da. für, daß $\beta$ die Positionen jeweils auch kompetent dargestellt werden. Zum 60.Jahrestag der Oktoberrevolution liegt damit eine umfassende und kritische Bilanz vor. ca. 300 Seiten, ca. DM 29.- ISBN 3921241278

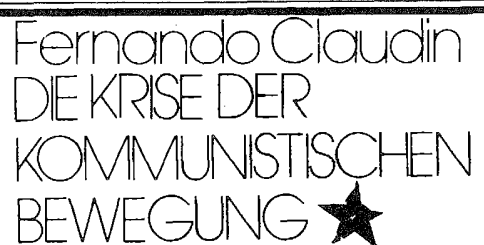

\section{VON DER KOMINTERN ZUR KOMINFORM}

Fernando Claudin hat in seinem umfassenden Werk als erster systematisch die Wechselbeziehungen zwischen dem Staatsinteresse der Sowjetunion und den sozial-revo lutionären Bewegungen in der ganzen Welt dargestellt. Er legt einen wissenschaftlich fundierten und zugleich flüssig lesbaren Text vor, der dem kritisch interessierten Leser einen hochinformativen Einstieg in den Problemkreis bietet.

Band I - Die Krise des internationalen Kommu nismus

Aus dem Inhalt: Auflösungstendenzen, Krise der Theorie, Monolithismus, Volksfrontexperimente u.v.a.

ca. 350 Seiten, ca, DM 25,- -erscheint im Juni Im Herbst erscheint:

Band II - Der Stalinismus auf dem Gipfel seiner Macht ca. 350 Seiten, ca. 25.- DM

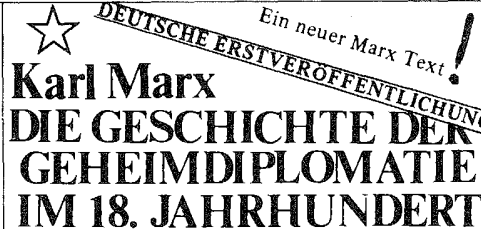

UBBER DEN ASIATISCHEN URSPRUNG DER RUSSISCHEN DESPOTIE

Die Brisanz des Marx-Textes und der darin enthaltenen Problemstellungen liegt darin, da $\beta$ in der scheinbar spezialisierten Diskussion über die Analyse der russischen Produk tionsverhältnisse und Überbauformen die Grundlagen für eine radikale Neuinterpretation der sowjetischen Übergang gesellschaft, des Stalinismus - samt seiner ideologischen Zersetzungs- und Abfollprodukte - gelegt werden, die uber eine ideologische Position hinausgeht und zu der Frage vorstößt, ob nicht in den russischen Produktionsverhältnissen und ihrer Einbettung in den Weltmarktzusammenhang die Tendenz zu Formen der okonomischen und politischen Despotie angelegt ist.

Mit einem ausfürlichen Kommentar von D.B. Rjasanov und einer Einleitung von Bernd Rabehl. 244 Seiten, DM 25.jetzt lieferbar

\section{ITEINA MERIK HANDBUCH 1}

간 Zur kapitalistischen Entwicklung 危 und politischen Repression

Hrsg.: V.Bennholdt-Thomsen, T.Evers, K.Meschkat, C.und U.Müller-Plantenberg, W.Olle und W.Schöller

Informationen, kritische Analysen und Diskussionen in. und ausländischer Autoren stehen im Mittelpunkt des er. sten Handbuches. In Länderberichten werden Grunddaten und ein $A$ briß der jüngsten politischen und wirtschaftlichen Entwicklungen der einzelnen Latein- und Mittelamerikani. schen Staaten gegeben.Daneben stehen detaillierte Studien uber die argentinischen Gewerkschaften,Arbeitslosigkeit $u$. Repression in Chile u.a.; analytische Beiträge zum Problem von Arbeitslosigkeit und Wirtschaftswachstum (Singer) und Diskussionen über die Unterentwicklung des Marxismus in Lateinamerika (Mires) u.v.a.

ca. 300 Seiten, ca. DM 22,- - erscheint im Juni

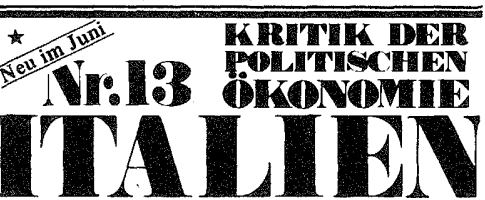

Dieses Heft behandelt zwei zentrale Aspekte der gegenwärtigen Situation Italiens: die politische Kräftekonstellation im Innern und die ökonomische Situation des Landes vor allem im Hinblick auf seine außenwirtschaft liche Abhängigkeit.

Beiträge von Krippendorf, Flores, Moretti, Claudin, u.a.

Einfachheft DM 6.-; Doppelheft DM 9.-; Dreifachheft DM 15.-; Abo, 4 Nr. incl. Porto DM 21.-

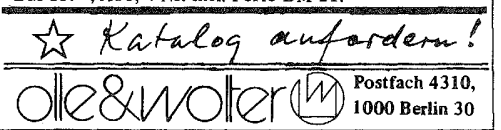

